\title{
Validity of cardiovascular magnetic resonance in pre- and post-operative evaluation of pulmonary arteries and ventricular functions in pediatric conotruncal anomalies
}

\author{
Samira Saraya ${ }^{1,2^{*}}$, Yousra Mahmoud Ahmad ${ }^{3}$, Hazem Hamed Soliman ${ }^{1}$, Mahmoud Saraya ${ }^{4}$ and Mariam Louis ${ }^{1}$
}

\begin{abstract}
Background: The aim of this work is to evaluate the validity of magnetic resonance (MR) imaging in assessment of pulmonary arteries and ventricular functions with conotruncal anomalies in the pediatric population.

Results: Between March 2018 and December 2019, 42 patients ranging in age from 6 months to 18 years and diagnosed with conotruncal anomalies by echocardiographic examination were submitted for cardiac MRI followed by assessment of their morphological (intra- and extra-cardiac anatomy) and functional parameters. The most common conotruncal anomaly was tetralogy of Fallot which represented $45 \%$ of the cases. Cardiac magnetic resonance (CMR) compared to echocardiography showed $46 \%$ agreement in the assessment of right ventricular volumes and function. There was only $37 \%$ agreement between echocardiography and MRI in delineation of MAPC AS.

Conclusion: CMR provides a powerful tool, giving anatomical and physiological information that echocardiography and catheterization alone cannot provide in conotruncal anomalies.
\end{abstract}

Keywords: Cardiac, Magnetic resonance, Conotruncal

\section{Background}

Conotruncal anomalies are a group of congenital heart defects that involve the outflow tracts of the heart and the great vessels [1].

It accounts for approximately $30 \%$ of the congenital heart disease being characterized by anomalies of the cardiac outflow tract or of the great arteries [2].

Developmental abnormalities of the embryonic conus arteriosus (infundibulum) and the truncus arteriosus result in abnormal ventriculo-arterial alignments and

\footnotetext{
* Correspondence: semsaraya@yahoo.com

${ }^{1}$ Faculty of Medicine, Cairo University, Kasr Al-Ainy, Cairo, Egypt

2Diagnostic and Intervention Radiology Department, Cairo University

Hospitals, El-Manial, Cairo 11956, Egypt

Full list of author information is available at the end of the article
}

connections, outlet septation defects, outlet hypoplasia, stenosis, or atresia [3].

The major conotruncal anomalies are tetralogy of Fallot (TOF), transposition of the great arteries (TGA), double-outlet right ventricle (DORV), truncus arteriosus, and interrupted aortic arch [1].

Echocardiography remains the first-line cardiovascular imaging modality in patients with congenital heart disease because of the portability, universal availability, being of low-cost, non-invasive with real-time assessment of the anatomy, function, and valvular diseases [4]. However, the suboptimal penetration of ultrasound poses the chief limitation of echocardiography, especially after cardiovascular surgery [5]. 
Where cardiac catheterization was traditionally used to provide hemodynamic information and visualize extracardiac great vessels, cardiovascular MR (CMR) is progressively fulfilling this role [5].

CMR provides a powerful tool in displaying extracardiac anatomy, including the great arteries, systemic, and pulmonary veins. Vascular and valvular flow can be assessed, shunts can be quantified, and myocardial function can be measured accurately with high reproducibility, regardless of ventricular morphology [6].

Finally, CMR surpasses both catheterization and echocardiography in providing high-resolution, isotropic, three-dimensional datasets [5].

The aim of this work is to evaluate the validity of magnetic resonance (MR) imaging in assessment of pulmonary arteries and ventricular functions with conotruncal anomalies in the pediatric population.

\section{Methods}

\section{Study population and design}

This is a retrospective comparative study between echocardiography and CMR conducted between March 2018 and December 2019 on 42 pediatric patients.

This study was approved by the research ethic committee of the Radiology Department of our university. All patients included in this study gave a written informed consent to participate in this research. If the patient was less than 16 years old or unconscious at the time of the study, written informed consent for their participation was given by their parent or legal guardian.

The patients' ages ranged from 6 months to 18 years (25 females and 17 males). All patients were submitted to both echocardiographic examination and cardiac MRI study.

\section{Echocardiography}

It was performed for all patients using the commercially available machines in the echocardiography unit and the cardiology department

\section{Magnetic resonance imager}

A Philips Gyroscan Intera (1.5 T) super conducting magnet was used.

\section{Patient selection and preparation Inclusion criteria}

Patients suspected clinically and diagnosed by echocardiography with conotruncal anomalies during the period of the study.

\section{Exclusion criteria}

Patients who have implanted electric and electronic devices and in particular; heart pacemakers (especially older types), insulin pumps, implanted hearing aids, neuro-stimulators, intracranial metal clips, and metallic bodies in the eye.

\section{Sedation}

Infants and small children up to the age of 4 years were sedated by oral chloral hydrate $(75 \mathrm{mg} / \mathrm{kg}$ body weight), while older children tolerated the examination without sedation. Infants and small children were wrapped in a blanket and placed on a thin, firm mattress. Instructions were given to the older children and adults to avoid any movement during the procedure.

\section{Patient position}

All patients were examined in supine position, head first. ECG leads and PEAR (phase encoding artifact reduction) sensors were applied. The infants utilized a $\mathrm{C} 1$ coil while older children used a synergy body coil.

\section{Imaging parameters}

The imaging protocol depended on the age of the patient and the congenital cardiac anomaly. The pediatric patient group was subjected to the conventional spin echo sequences (black blood technique) due to failure to execute breath hold as they were sedated. The older children were subjected to the conventional spin echo sequences (black blood technique) and fast gradient echo pulse sequences (bFFE/BH) balanced fast field echo/breath hold technique.

The three-dimensional (3D) whole heart approach with respiratory navigator gating and ECG triggering was used for comprehensive evaluation of thoracic vasculature to give detailed morphological information in conotruncal anomalies.

Ventricular volumes were calculated for all patients including; end diastolic volume, end systolic volume, stroke volume, ventricular ejection fraction, and cardiac output.

This was obtained through using ECG-gated steadystate free precession (SSFP) imaging sequences (TE 1.5$2.0 \mathrm{~ms}$, TR $2.8-4.0 \mathrm{~ms}$, flip angle $45^{\circ}$, views per segment 10-20, reconstructed images per cardiac cycle 20-30) where a stack of 12 contiguous short-axis slabs perpendicular to the long axis of ventricles (slice thickness 6-8 $\mathrm{mm}$, interslice distance $0-2 \mathrm{~mm}$ ) was obtained assuring full coverage of ventricles.

A commercially available computer workstation (ViewForum, Philips Medical Systems, Best, The Netherlands) was used for image processing. Cross referencing of the long- and short-axis cine images of the ventricles facilitated identification of the required ventricular function parameters to be included in the analysis 


\section{Image interpretation}

Ventricular volumes were calculated using manual contour tracing of short-axis slices from the apex to the base to obtain end-diastolic and end-systolic volumes.

The stroke volume (SV) was calculated by subtracting the end systolic volume (ESV) from the end diastolic volume (EDV). The ejection fraction was calculated by dividing the SV by the EDV.

The anatomical and functional data were correlated with the echocardiographic findings.

\section{Cardiac catheterization}

It was performed for selected number of patients (7 cases) using the available machines in the cardiac catheterization units in the children hospital.

\section{Statistical method}

The collected data were coded, tabulated, and statistically analyzed using IBM SPSS statistics (Statistical Package for Social Sciences) software version 18.0, IBM Corp., Chicago, USA, 2009.

\section{Results}

This study included 42 patients with 25 males $(60 \%)$ and 17 females (40\%) with an age range of 6 months-18 years (mean age 6.3).

Twenty-seven of the patients were newly diagnosed with conotruncal heart defects (64\%) while the remaining $15(36 \%)$ were operated upon and came for post-operative evaluation.

The most common conotruncal anomaly was tetralogy of Fallot which represented $45 \%$ of the cases while the least common was double outlet right ventricle representing $7 \%$ of the cases. Others were L-TGA: $26 \%$ and D-TGA: $22 \%$

In this study, the results were classified according to the patients' pathological finding as follows:

(1) Pulmonary artery anomalies.

(2) Aorto-pulmonary collaterals.

(3) Assessment of right ventricular volumes and functions

(4) Assessment of post-operative cases

\section{Pulmonary artery anomalies}

Eighteen of the patients in our study had a variable degree of pulmonary artery anomalies. Delineation of the pulmonary arterial tree abnormalities were performed by echocardiography and MRI in preoperative cases (Figs. 1 and 2). Points of consideration in the evaluation in conotruncal anomalies included pulmonary atresia, confluent and non-confluence of the pulmonary arteries, pulmonary stenosis, and pulmonary agenesis (Table 1). Non-visualization of the pulmonary artery especially the left pulmonary artery (LPA) was considered a considerable limitation of echocardiography and hence the superiority of MRI in delineation of the pulmonary arterial tree.

The echo failed to detect eight of the pulmonary artery anomalies. Those false-negative cases in which the pulmonary arterial tree could not be visualized by echocardiography were due to various reasons including technical difficulties during performing the study, nonconfluent pulmonary arteries, and LPA agenesis (Fig. 3).

\section{Aorto-pulmonary collaterals}

Eight of the examined cases had major aorto-pulmonary collateral arteries (MAPCAS) detected by MRI, while echo failed to detect five patients out of them (Fig. 4).

According to the above results, there was only 37\% agreement between echocardiography and MRI in delineation of MAPCAS which is of great importance in preoperative evaluation of these patients. The technique used for MR evaluation of these patients was conventional spin echo, cine images, and the $3 \mathrm{D}$ navigator. These techniques delineated the MAPCAS supplying the lungs but neither the lobar nor the segmental distribution of those MAPCAS was delineated by conventional spin echo. The difficulties with tracking vessels after they entered the lung parenchyma resulted from their decreasing size as they arborized, and a lower contrast to noise ratio in the lung tissue.

\section{Assessment of right ventricular volumes and functions}

Right ventricular end-diastolic and end-systolic volumes, mass, stroke volumes, and ejection fractions were measured in 37 of our patients. Measurements were made by a radiographer blinded to the echocardiographic data (Fig. 5). Based on the published normal values for right ventricular ejection fraction (RVEF), in this study, we followed Andrew $\mathrm{M}$ et al. 2011 in their classification as they roughly defined a RVEF of $40-50 \%$ as mildly decreased RV function, and RVEF of 30 $40 \%$ as moderately decreased RV function, and RVEF $<30 \%$ severely decreased RV function.

Compared to echocardiography for assessment of right ventricular volumes and functions, CMR was preferred for its ability to overcome the underestimation of the echocardiography to those measurements as echocardiography significantly underestimated volumes in the 17 patients as a whole. Mean RV end diastolic volumes were significantly greater when measured by CMR compared to echocardiography with $46 \%$ agreement.

\section{Assessment of post-operative cases} Pulmonary regurge in patients with repaired TOF

Six of the TOF cases came for post-operative evaluation to assess the degree of pulmonary regurge (PR). This is shown in Fig. 6 in details. 


\section{Case 1:}

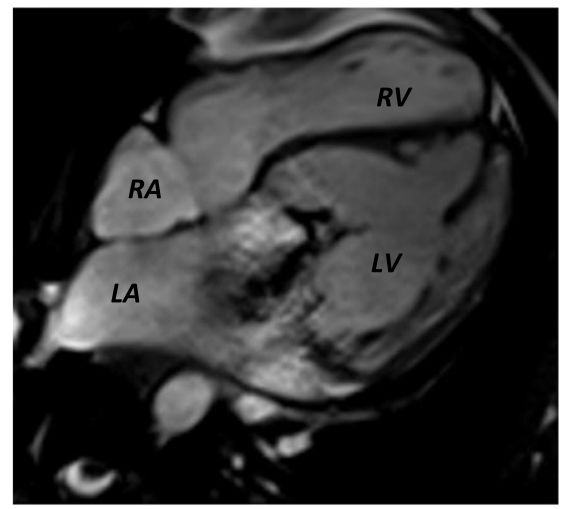

(a)

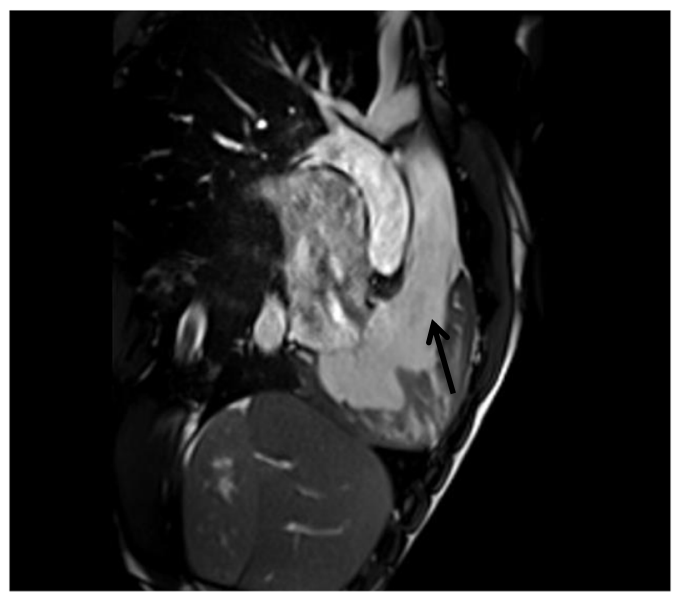

(c)

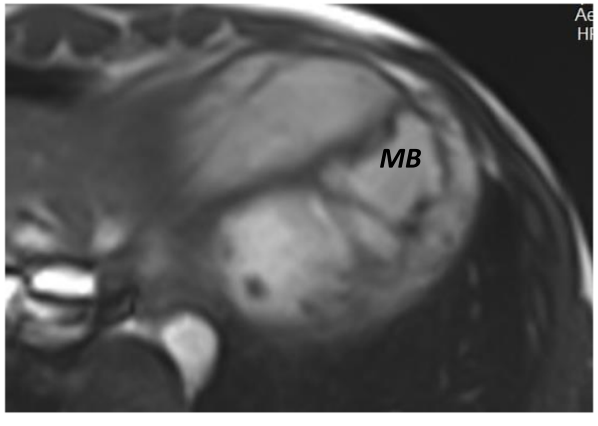

(b) morphologic LV and the left atrium connected to a trabeculated, left-sided, morphologic RV. The mitral valve displaced more basally than the tricuspid valve. a The image shows the moderator band (MB) of the left sided morphologically right ventricle (b). c Right ventricular outflow tract showing the regurgitant jet at the tricuspid valve region (arrow)

According to our results, we discovered that echocardiography was less sensitive in the exact estimation of the pulmonary regurge in cases when the regurge was less than $20 \%$ as it overestimated regurgitation fraction (RF) resulting in false-positive results, e.g., two cases were diagnosed as moderate PR by echocardiography while the MRI proved that the RF was $<20 \%$, in contrary, echocardiography was sensitive in pulmonary regurge $>20 \%$ as CMR.

\section{Assessment of post-operative complications in patients with transposition of great vessels}

Eight of the patients with TGA came for post-operative evaluation. Magnetic resonance imaging and echocardiography were compared for their ability to demonstrate the post-operative complications. MRI detected that three cases of them with left pulmonary stenosis (37.5\%) and one case with left ventricular outflow obstruction $(12.5 \%)$ and one case with severe pulmonary arterial dilatation to the extent of pulmonary hypertension (12\%). Echocardiography failed to show two cases with left pulmonary stenosis due to poor acoustic window.

\section{Other anomalies were detected as follows \\ L-TGA}

Levo-transposition of the great arteries (L-TGA), also called congenitally corrected TGA, characterized by atrioventricular and ventriculoarterial discordance was detected in $26 \%$ of the patients.

\section{$D-T G A$}

Dextro-transposition of the great arteries characterized by ventriculo-arterial discordance was detected in $22 \%$. 


\section{Case 2}

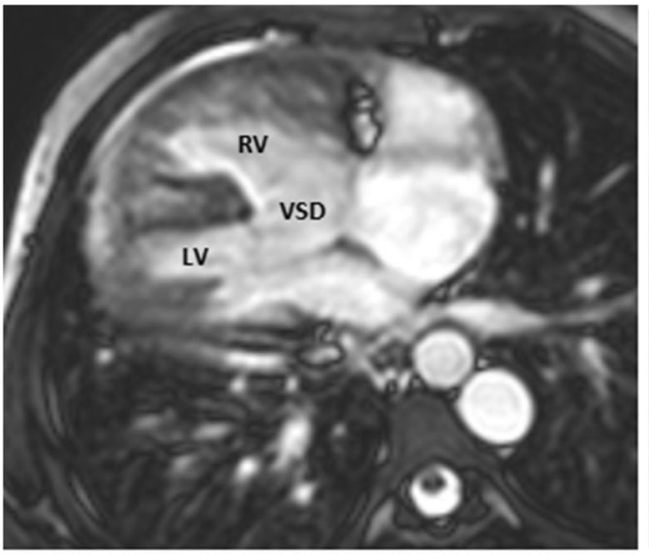

(a)

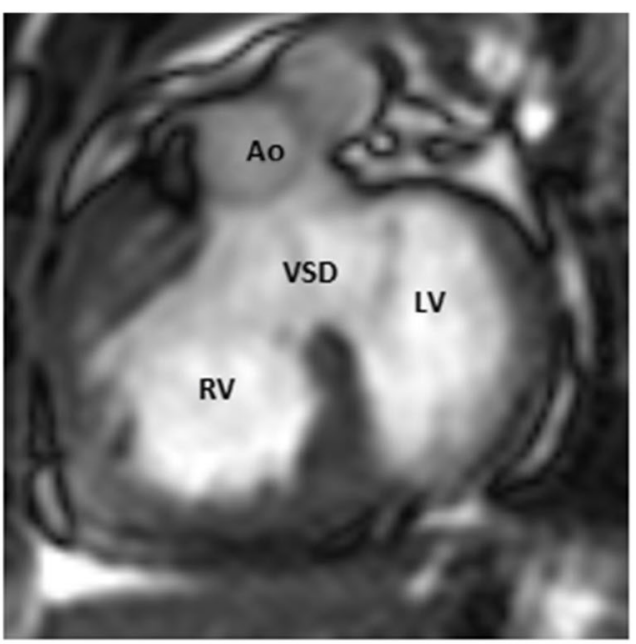

(c)

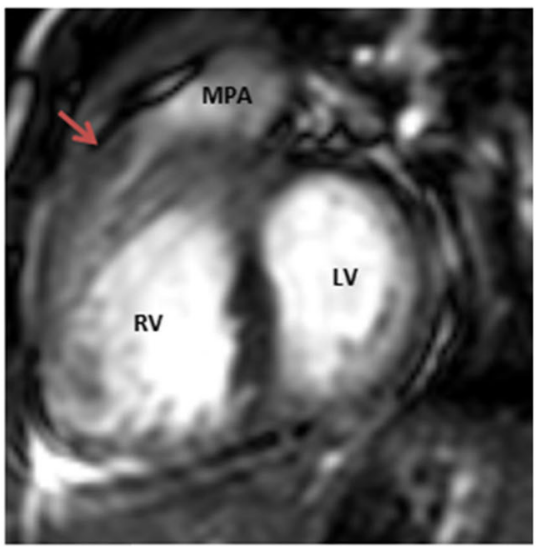

(b)

Fig. 2 A case of pre-operative TOF. cine MRI images showing a axial view showing Dextrocardia with right ventricular hypertrophy. b SA view showing infundibular pulmonary stenosis "red arrow". c SA view showing subaortic VSD

\section{DORV}

Double outlet right ventricle in which both great arteries arise completely or predominantly from the right ventricle represented $7 \%$ of the patients.

\section{Discussion}

Cardiac and vascular anatomy of patients with congenital heart disease (CHD) is complex and accurate evaluation mandates MR image acquisition in several nonstandard planes. The most important sequences for anatomical imaging in CHD are black-blood spin echo, 2D SSFP, and 3D SSFP techniques as well as contrastenhanced MR angiography [6].

Greil et al. 2015 reported that the CMR is the current clinical gold standard for measurements of ventricular function and blood flow which are crucial components of a CMR examination in patients with CHD. Ventricular volumes and ejection fraction are assessed by acquisition of a stack of SSFP cine slices covering the entire heart [7].

In this study, the role of cardiac magnetic resonance imaging in the evaluation of conotruncal anomalies was assessed. The study enrolled 42 patients (25 males and 17 females) with an age range between 6 months and 18 years. All patients were at least subjected to one echocardiographic examination while seven of them underwent cardiac catheterization.

The most common conotruncal anomaly in our study was tetralogy of Fallot which represented $45 \%$ of the patients while the least common was double outlet right ventricle which represented only $7 \%$ of them. The primary goal of the cardiovascular MR imaging 
Table 1 Number of patients according to type of pulmonary artery abnormality by echocardiography and MRI

\begin{tabular}{lllll}
\hline Abnormality & Echo & \% & MRI & $\%$ \\
\hline Atresia & 6 & 33.3 & 9 & 50 \\
Non-confluent & 2 & 11.1 & 5 & 27.8 \\
Stenosis & 2 & 11.1 & 4 & 22.2 \\
\hline
\end{tabular}

examination of the patients in our study is to assess the full anatomical details of the patients pre-operatively and to follow-up the patients post-operatively for early detection of complications.

Cardiac MRI detected 18 patients in this study with variable degrees of pulmonary artery stenosis ranging from atresia to mild stenosis. While echocardiography failed to detect eight of them due to left pulmonary artery agenesis, non-confluence of the pulmonary artery branches and due to technical problems. The agreement between CMR and echocardiography was $44 \%$. Cardiac MRI also successfully detected eight of the aortopulmonary collaterals with $37 \%$ agreement with echocardiography as the latter did not visualize five of them.

In our study, no contrast-enhanced magnetic resonance angiography (CE-MRA) was used for the patients in our study because no bolus tracking software for delineation of pulmonary vasculature was available and manual injection of contrast with the rapid heart rate of the pediatric group of patients makes it difficult to assess pulmonary vessels without overlap of aortic and systemic vessels.

We acknowledge that the lack of utilization of contrast medium in our study as a limitation which probably

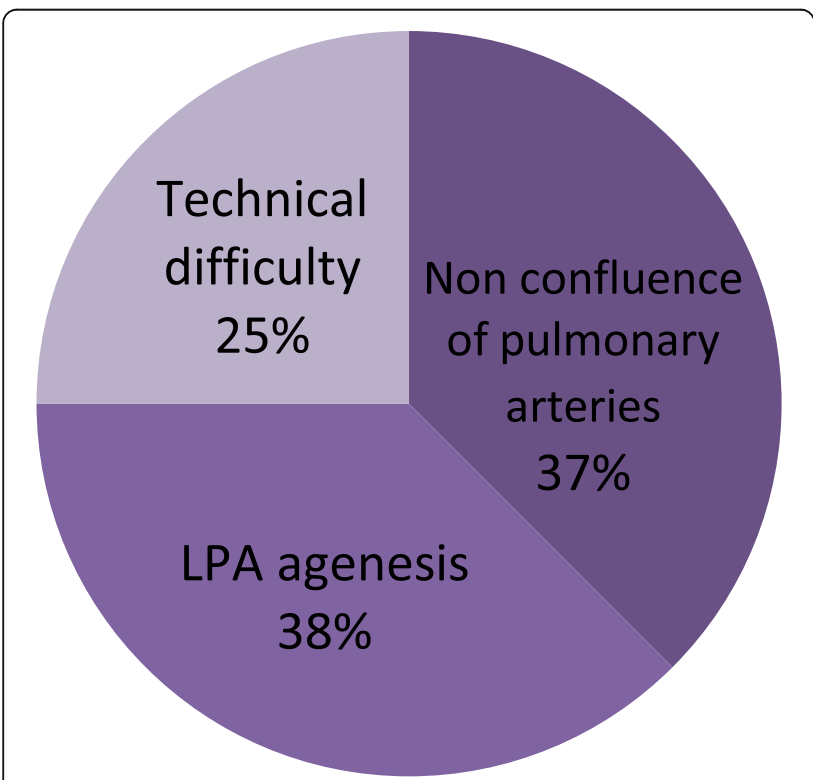

Fig. 3 Causes of non-visualization of the pulmonary arterial tree by echocardiography as a percentage reduced the sensitivity of the examination in anatomical measurements of the vascular structures. Büchel et al. 2005 stated that CE-MRA provides accurate quantitative anatomical information, which highly agrees with conventional angiographic data, and can therefore be used for planning catheter-guided procedures [8].

However, free-breathing whole-heart 3D SSFP or black-blood fast spin-echo sequence was efficient for visualization of the atretic/hypoplastic pulmonary arteries and visualization of the aorto-pulmonary collaterals. In our study, the size of the hypoplastic pulmonary arteries is well measured. The origin of the coronary arteries was seen as well.

This study enrolled 9 patients with D-TGA and 11 with L-TGA. Cardiac MRI played an important role in anatomical and functional assessment of those patients. This agreed with Fogel et al. 2010 which reported that CMR has been recognized as an accurate non-invasive imaging tool to diagnose and evaluate patients with TGA as it shows the discordant atrioventricular and ventricular connections which can be visualized by identifying the right atrial (triangular atrial appendage with wide ostium) and left atrial (tubular atrial appendage with narrow ostium) anatomy and situs, and biventricular as well as large vessel anatomy with the aorta anterior and to the left, and can also show the great arteries side-by-side. Also, details of AV valve anatomy may be visualized [9].

Assessment of right ventricular volumes and functions was an important issue in our study as the conotruncal anomalies affects them in different degrees. Thirty-seven of our patients were subjected to right ventricular volumes and functions measurements. They show 46\% agreement with echocardiography as echocardiography significantly underestimated volumes in the 17 patients as a whole and the mean right ventricular end diastolic volumes (RVED) were significantly greater when measured by CMR. Furthermore, CMR was superior to echocardiography for the assessment of the stroke volume and ejection fraction of the right ventricle. Minor early changes in ventricular size and function are more readily detectable, given the reproducibility of CMR, and may lead to an early appropriate medical intervention

This was agreed with Andrew M et al. 2011 as they reported that 3D-Echo systematically underestimates right ventricular volumes compared to cardiovascular magnetic resonance in adult congenital heart disease patients with moderate or severe RV dilatation [10].

Geva et al. 2014 also concluded that CMR is presently the best diagnostic modality for assessment of RV size and function in patients with CHD. Furthermore, a growing literature informs clinicians on how to use CMR data to guide patient management. Echocardiography, which is more widely available, provides useful 


\section{MAPCAs}

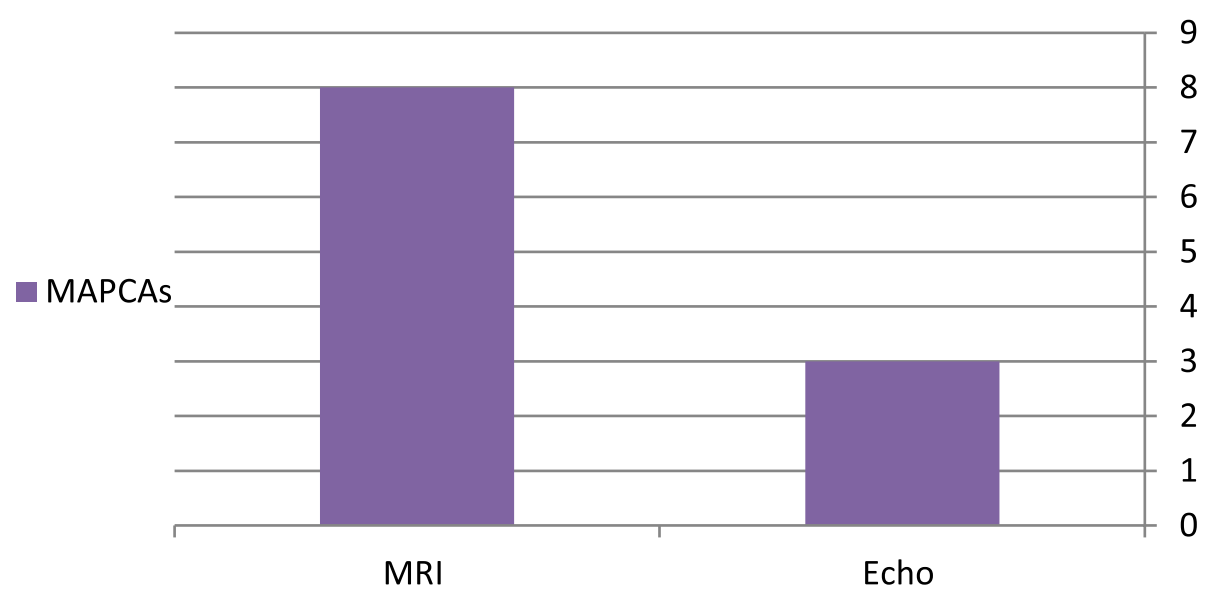

Fig. 4 Number of cases of aorto-pulmonary connection by echocardiography and MRI. *MAPCAs: Major aorto-pulmonary collateral arteries

diagnostic information in many clinical circumstances that affect the right heart. However, when precise quantitative data are required to make important clinical decisions (e.g., when to recommend pulmonary valve replacement), CMR remains the diagnostic modality of choice. As new echocardiographic, CMR, and other imaging techniques continue to evolve, it would be interesting to revisit this controversy in the future [11].

Our results agreed with Laura et al. 2012; their study concluded that in correlation between CMR and echocardiography, the latter overestimated the pulmonary regurge when the $\mathrm{RF}<20 \%$ resulting in a false-positive results of about $37 \%$ [12].

In this study, regarding the post-operative evaluation for the patients with transposition of great vessels, eight of the cases came for post-operative follow-up.

CMR quantified blood flow through valves and vessels and provided valuable information concerning outflow tract stenosis and regurgitation fractions that can be

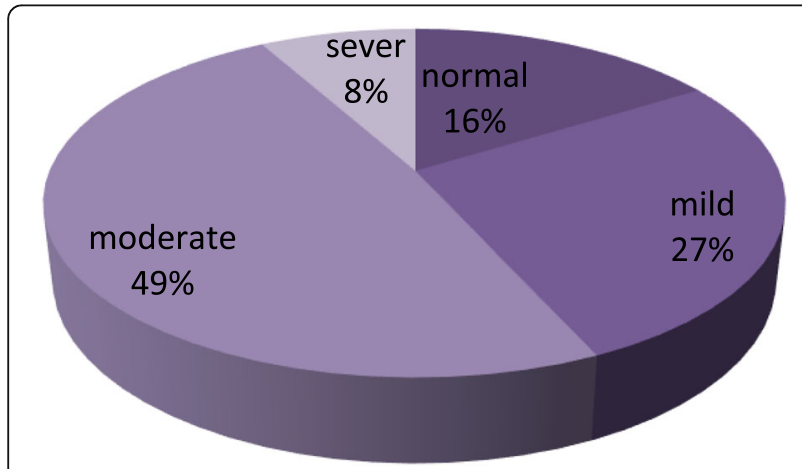

Fig. 5 Percentage of patients according to the degree of right ventricular dilatation by MRI followed over time and aid in management decisions. MRI can also depict the abnormal position of the great arteries, with the aorta anterior to the pulmonary artery and arising from the right ventricle in cases of $\mathrm{D}$ transposition of great arteries and double outlet right ventricle with $\mathrm{D}$-malposed vessels.

CMR was a very important tool in evaluation of postoperative complications in those patients. The postoperative evaluation requires a thorough evaluation of the right ventricular function and possible tricuspid valve regurgitation, left ventricular outflow tract

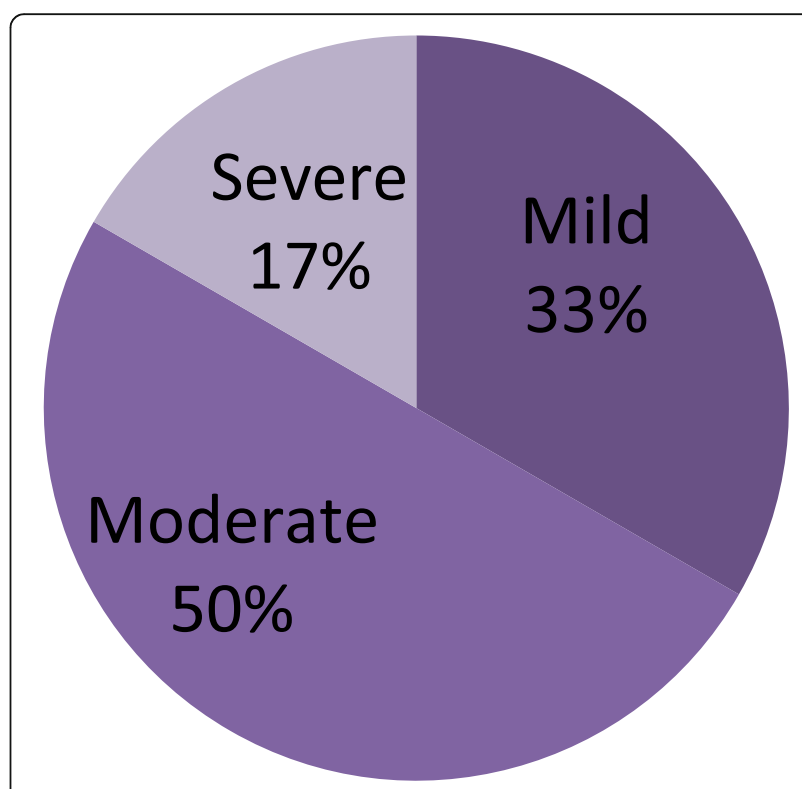

Fig. 6 Percentage of patients according to the degree of PR in postTOF repair by MRI. *PR: pulmonary regurge, TOF: tetralogy of Fallot 
obstruction, arrhythmias, baffle leaks, and systemic and pulmonary venous pathway obstruction.

Adam et al. 2012 studied the evaluation with cardiovascular MR imaging of baffles and conduits used in palliation or repair of congenital heart disease and concluded that CMR imaging is the imaging modality of choice for evaluation of baffles and conduits used in repair or palliation of complex congenital heart disease as it provides a non-invasive assessment of anatomy, function, and flow without the use of ionizing radiation [13].

Sangeeta et al. 2015 studied managing heart failure in transposition of the great arteries and reported that although the right ventricle is difficult to evaluate because of its complex shape, cardiac MRI is the gold standard of right ventricular assessment because of its ability to completely visualize the right ventricle. It is not only used to quantify the size and function of the right ventricle but also to identify associated structural abnormalities and areas of concern after surgery, such as aortic root, branches of pulmonary arteries, and right ventricular outflow tract [14].

One of the patients in our study with D-TGA who underwent surgical intervention presented with symptoms of pulmonary arterial hypertension. CMR revealed marked dilatation of the pulmonary arteries. Pulmonary arterial hypertension which is a rare complication has been reported also by Janani et al. 2017 and reported that lateonset pulmonary arterial hypertension (PAH) is a rare but fatal complication in patients with childhood surgical repair of dextro-transposition of great arteries (D-TGA). The pathogenic mechanisms of pulmonary arterial hypertension in patients with repaired D-TGA are not well understood and treatment is not standardized [15].

\section{Conclusion}

Our study concluded that CMR provides a powerful tool, giving anatomical and physiological information regarding the pulmonary arteries and ventricular functions in pediatric conotruncal anomalies as it overcomes many of the limitations of echocardiography (e.g., restricted acoustic windows), computed tomography (e.g., exposure to ionizing radiation, lack of functional information), and cardiac catheterization (e.g., ionizing radiation exposure and morbidity). CMR is also the diagnostic modality of choice in giving precise quantitative data that are required to make important clinical decisions (e.g., when to recommend pulmonary valve replacement in patients with repaired TOF).

\section{Abbreviations}

MR: Magnetic resonance; TOF: Tetralogy of Fallot; DORV: Double-outlet right ventricle; CMR: Cardiovascular magnetic resonance; ECG: Electrocardiogram; ECHO: Echocardiography; PEAR: Phase encoding artifact reduction; 2D : 2dimensional; 3D : 3-dimensional; bFFE/BH: Balanced fast field echo/breath hold; MAPCAS : Major aorto-pulmonary collateral arteries; MRI: Magnetic resonance imaging; RV: Right ventricle; RVEF: Right ventricular ejection fraction; RF: Regurgitation fraction; PR: Pulmonary regurge; TGA: Transposition of the great arteries; CHD: Congenital heart disease: SSFP: Steady state free precession; CEMRA: Contrast-enhanced magnetic resonance angiography; RVEDV: Right ventricular end diastolic volume; LPA: Left pulmonary artery

\section{Acknowledgements}

Not applicable

\section{Authors' contributions}

Samira Saraya (SS), Yousra Gwaily (YG), Hazem Hamed Soliman (HHS), Mahmoud Saraya (MS), and Mariam Louis (ML) contributed to this work. ML and SS designed the research. ML, MS, YG, and SS performed research. HHS, SS, YG, MS, and ML analyzed the data. HHS, SS, ML, and MS wrote the paper. All authors have read and approved the manuscript.

\section{Funding}

The study has no funding from any resource.

Availability of data and materials

The datasets used and/or analyzed during the current study are available from the corresponding author on reasonable request.

\section{Declarations}

Ethics approval and consent to participate

This study was approved by the research ethic committee of the Radiology Department of the Faculty of Medicine at Cairo University on 08 January 2018; reference number of approval: 894-2017.

All patients included in this study gave a written informed consent to participate in this research. If the patient was less than 16 years old or unconscious at the time of the study, written informed consent for their participation was given by their parent or legal guardian.

\section{Consent for publication}

All patients included in this study gave a written informed consent to publish the data contained within this study. If the patient was less than 16 years old or unconscious at the time of the study, written informed consent for publication was given by their parent or legal guardian.

\section{Competing interests}

The authors declare that they have no competing interests.

\section{Author details}

${ }^{1}$ Faculty of Medicine, Cairo University, Kasr Al-Ainy, Cairo, Egypt. ${ }^{2}$ Diagnostic and Intervention Radiology Department, Cairo University Hospitals, El-Manial, Cairo 11956, Egypt. ${ }^{3}$ National Heart Institute, Cairo, Egypt. ${ }^{4}$ Faculty of

Medicine, Cardiology Department, Cairo University, Kasr Al-Ainy, Cairo, Egypt.

Received: 20 October 2020 Accepted: 10 May 2021

Published online: 22 May 2021

\section{References}

1. Frank L, Dillman JR, Parish V, Mueller GC, Kazerooni EA, Bell A, Attili AK (2010) Cardiovascular MR imaging of conotruncal anomalies 1. Radiographics. 30(4):1069-1094. https://doi.org/10.1148/rg.304095158

2. Amodeo S, Vitrano G, Guardino M, Paci G, Corselli F, Antona V, Barrano G, Magliozzi M, Novelli A, Venezia R, Corsello G (2020) What is the impact of a novel MED12 variant on syndromic conotruncal heart defects? Analysis of case report on two male sibs. Ital J Pediatr 6(1):1-6

3. Saremi F, Ho SY, Cabrera JA, Snchez-Quintana DN (2013) Right ventricular outflow tract imaging with $C T$ and MRI: part 1, morphology. Am J Roentgenol 200(1):W39-W50. https://doi.org/10.2214/AJR.12.9333

4. Muscogiuri G, Suranyi P, Eid M, Varga-Szemes A, Griffith L, Pontone G, Schoepf UJ, De Cecco CN (2019) Pediatric cardiac MR imaging:: practical preoperative assessment. Magn Reson Imaging Clin 27(2):243-262, Pediatric Cardiac MR Imaging. https://doi.org/10.1016/j.mric.2019.01.004

5. Ntsinjana HN, Hughes ML, Taylor AM (2011) The role of cardiovascular magnetic resonance in pediatric congenital heart disease. J Cardiovasc Magn Reson 13(1):51. https://doi.org/10.1186/1532-429X-13-51 
6. Giuseppe M, Secinaro A, Ciliberti P, Fuqua M, Nutting A (2017) Utility of cardiac magnetic resonance imaging in the management of adult congenital heart disease. J Thorac Imaging 32(4):233-244

7. ValsangiacomoBuechel ER, Grosse-Wortmann L, Fratz S, Eichhorn J, Sarikouch S, Greil GF, Beerbaum P, Bucciarelli-Ducci C, Bonello B, Sieverding L, Schwitter J (2015) Indications for cardiovascular magnetic resonance in children with congenital and acquired heart disease: an expert consensus paper of the Imaging Working Group of the AEPC and the Cardiovascular Magnetic Resonance Section of the EACVI. Eur Heart I Cardiovasc Imaging 16(3):281-297. https://doi.org/10.1093/ehjci/jeu129

8. Büchel ER, DiBernardo S, Bauersfeld U, Berger F (2005) Contrast-enhanced magnetic resonance angiography of the great arteries in patients with congenital heart disease: an accurate tool for planning catheter-guided interventions. Int J Cardiovasc Imaging 21(2-3):313-322. https://doi.org/10.1 007/s10554-004-4017-y

9. Fogel MA. editor. Principles and practice of cardiac magnetic resonance in congenital heart disease: form, function and flow. Wiley; 2010. ISBN: 978-1444-31704-6.

10. Crean AM, Maredia N, Ballard G, Menezes R, Wharton G, Forster J, Greenwood JP, Thomson JD (2011) 3D Echo systematically underestimates right ventricular volumes compared to cardiovascular magnetic resonance in adult congenital heart disease patients with moderate or severe RV dilatation. J Cardiovasc Magn Reson 13(1):78. https://doi.org/10.1186/1 532-429X-13-78

11. Geva T (2014) Is MRI the Preferred Method for Evaluating Right Ventricular Size and Function in Patients With Congenital Heart Disease? Circ Cardiovasc Imaging 7(1):190-197. https://doi.org/10.1161/CIRCIMAGING.113. 000553

12. Mercer-Rosa L, Yang W, Kutty S, Rychik J, Fogel M, Goldmuntz E (2012) Quantifying pulmonary regurgitation and right ventricular function in surgically repaired tetralogy of Fallot: a comparative analysis of echocardiography and magnetic resonance imaging. Circ Cardiovasc Imaging 5(5):637-643

13. Lu JC, Dorfman AL, Attili AK, GhadimiMahani M, Dillman JR, Agarwal PP (2012) Evaluation with cardiovascular MR imaging of baffles and conduits used in palliation or repair of congenital heart disease. Radiographics. 32(3): E107-E127. https://doi.org/10.1148/rg.323115096

14. Shah S, Gupta T, Ahmad R (2015) Managing Heart Failure in Transposition of the Great Arteries. Ochsner J 15(3):290-296

15. Baskaran J, March KS, Thenappan T (2017) Late-onset pulmonary arterial hypertension in repaired D-transposition of great arteries: an uncommon complication. Pulm Circ 7(1):2045893217699228

\section{Publisher's Note}

Springer Nature remains neutral with regard to jurisdictional claims in published maps and institutional affiliations.

\section{Submit your manuscript to a SpringerOpen ${ }^{\circ}$ journal and benefit from:}

- Convenient online submission

- Rigorous peer review

- Open access: articles freely available online

- High visibility within the field

- Retaining the copyright to your article

Submit your next manuscript at $\boldsymbol{\nabla}$ springeropen.com 